DOI: $10.15393 /$ j3.art.2018.5410

The paper is presented at the conference "Complex analysis and its applications" (COMAN 2018), Gelendzhik - Krasnodar, Russia, June 2-9, 2018.

UDC $517.53 / .55$

A. A. Tatarkin, U. S. Saranchuk

\title{
ELEMENTARY SOLUTIONS OF A HOMOGENEOUS q-SIDED CONVOLUTION EQUATION
}

\begin{abstract}
Spectral synthesis on the complex plane related to solutions of some homogeneous equations of convolution type. There is a method to obtain solutions: we describe the elementary solutions set of the equation (spectral analysis) and prove the approximation theorem (spectral synthesis). In this paper we use the method for some homogeneous equations of convolution type, which appears from spectral synthesis problem for some differential operator.
\end{abstract}

Key words: differential operator, holomorphic function, spectral synthesis, spectral analysis, shift operator, convolution equation

2010 Mathematical Subject Classification: $34 \mathrm{LO5}$

1. Introduction. Let $\Omega_{0}, \Omega$ be convex domains in $\mathbf{C}, \varepsilon>0$, $U_{\varepsilon}=\{z:|z|<\varepsilon\}$. Suppose that $\Omega_{0}+U_{\varepsilon} \subseteq \Omega ; O(\Omega), O\left(U_{\varepsilon}\right), O\left(\Omega_{0}\right)$ and $O(\mathbf{C})$ are spaces of holomorphic functions equipped with the topology of uniform convergence on compact sets. The operator $D$ takes a function $f$ to $f^{\prime}$. The operator $D^{q}$ takes a function $f$ to $f^{(q)}$. Consider a shift operator

$$
T_{h}: f(z) \rightarrow f(z+h)
$$

where $h \in U_{\varepsilon}$ is the shift. The operator takes $O(\Omega)$ to $O\left(\Omega_{0}\right)$ and is continuous. The shift operator $T_{h}$ can be identified with the infinite order differential operator $\exp h D$. The characteristic function of the operator $T_{h}=\exp h D$ is equal to $\exp h \lambda$, we have

$$
\frac{T_{h}(\exp \lambda z)}{\exp \lambda z}=\exp h \lambda .
$$

(C) Petrozavodsk State University, 2018

\section{$(\mathrm{cc})$ EY-NC}


Let $q$ be a natural number, $a_{0}, \ldots, a_{q-1}$ be a set of complex numbers containing a non-zero one. Denote by $\omega_{q}$ the number $\exp \frac{2 \pi i}{q}$. Let $A$ be a continuous linear operator that acts on elements of the space of entire functions $O(\mathbf{C})$. Define $A$ by the rule

$$
g(\lambda) \rightarrow \sum_{k=0}^{q-1} a_{k} g\left(\omega_{q}^{k} \lambda\right),
$$

$A T_{h}$ is a continuous linear operator that takes $O(\Omega)$ to $O\left(\Omega_{0}\right)$ by the rule

$$
f(z) \rightarrow \sum_{k=0}^{q-1} a_{k} f\left(z+\omega_{q}^{k} h\right) .
$$

The operator $A T_{h}: O(\Omega) \rightarrow O\left(\Omega_{0}\right)$ is called a $q$-sided shift operator ( $h \in U_{\varepsilon}$ is the shift). Choose a continuous linear operator $S$ on the space $O\left(\Omega_{0}\right)$ and an arbitrary $q$-sided shift operator $A T_{h}: O(\Omega) \rightarrow O\left(\Omega_{0}\right)$. The equation

$$
\left\langle S, A T_{h}(f)\right\rangle=0, f \in O(\Omega),
$$

is called a homogeneous q-sided convolution equation. The solution space of a homogeneous $q$-sided convolution equation is equal to the kernel of the respective $q$-sided convolution operator $O(\Omega) \rightarrow O\left(U_{\varepsilon}\right) \mid f \rightarrow\left\langle S, A T_{h}(f)\right\rangle$. This space is a closed subspace in the space $O(\Omega)$ and is an invariant set for the differential operator $D^{q}$ [3]. Any exponential polynomial is called an elementary solution if this exponential polynomial satisfies the condition (1).

Let $q=1, \varphi(h)=\langle S, \exp h z\rangle$ be the characteristic function of the functional $S$. Suppose that $\lambda$ is a zero of the function $\varphi$ that has multiplicity $n$. Then exponential monomials of the form:

$$
\exp \lambda z, z \exp \lambda z, \ldots, z^{n-1} \exp \lambda z
$$

satisfy the condition (1) and are in $W_{S}$. It is well known that any exponential monomial has the form (2) if this monomial satisfies the condition (1). It follows that any elementary solution of the equation (1) is a linear combination of monomials of the form (2), where $\lambda \in C$ is a zero of the characteristic function of the analytic functional $S$ and $n$ is the zero's multiplicity $[1,2]$.

Choose $q \geqslant 1$; then we obtain a more complicated description of the solution sets of homogeneous $q$-sided convolution equations. The spectral 
synthesis problem for the differential operator $D^{q}$ appears; it requires the description of the differential operator's root vectors that are in a $D^{q}$ - invariant set $W \subseteq O(\Omega)[3]$.

\section{Properties of a $q$-sided shift operator.}

Property 1. A q-sided shift operator $A T_{h}$ can be identified with the infinite order differential operator

$$
f(z) \rightarrow \sum_{n=0}^{\infty} b_{n} \frac{h^{n}}{n !}\left(D^{n} f\right)(z)
$$

where

$$
b_{n}:=\sum_{k=0}^{q-1} a_{k} \omega_{q}^{k n}, n \in\{0,1, \ldots\}
$$

and the series (3) uniformly converges on each compact subset of $\Omega_{0}$.

Proof. Suppose that $f \in O(\Omega)$. By definition we have:

$$
A T_{h}(f)(z)=\sum_{k=0}^{q-1} a_{k} f\left(z+\omega_{q}^{k} h\right)=\sum_{k=0}^{q-1} a_{k}\left(\exp \omega_{q}^{k} h D\right)(f)(z),
$$

where $z \in \Omega_{0}$.

By the operator's $\exp \omega_{q}^{k} h D$ definition we have:

$$
\begin{gathered}
A T_{h}(f)(z)=\sum_{k=0}^{q-1} a_{k} \sum_{n=0}^{\infty} \frac{\omega_{q}^{k n} h^{n}}{n !}\left(D^{n} f\right)(z)= \\
=\sum_{n=0}^{\infty}\left(\sum_{k=0}^{q-1} a_{k} \omega_{q}^{k n}\right) \frac{h^{n}}{n !}\left(D^{n} f\right)(z)=\sum_{n=0}^{\infty} b_{n} \frac{h^{n}}{n !}\left(D^{n} f\right)(z) .
\end{gathered}
$$

For any $k \in\{0,1, \ldots, q-1\}$ the series

$$
\sum_{n=0}^{\infty} \frac{\omega_{q}^{k n} h^{n}}{n !}\left(D^{n} f\right)(z)
$$

uniformly converges on each compact subset of $\Omega_{0}$ to the function $f(z+$ $\left.+\omega_{q}^{k} h\right)$. Then the series (3) is uniformly convergent on compact subsets of $\Omega_{0}$ too. 
Property 2. The characteristic function of a q-sided shift operator $A T_{h}: O(\Omega) \rightarrow O\left(\Omega_{0}\right)$ is equal to the function

$$
A(\exp h \lambda):=\sum_{k=0}^{q-1} a_{k} \exp \omega_{q}^{k} h \lambda=\sum_{n=0}^{\infty} b_{n} \frac{h^{n} \lambda^{n}}{n !},
$$

then

$$
\frac{A T_{h}(\exp \lambda z)}{\exp \lambda z}=A(\exp h \lambda) .
$$

Proof. On the one hand, by definition of the $q$-sided shift operator for any $z \in \Omega_{0}$ we have

$$
A T_{h}(\exp \lambda z)=\sum_{k=0}^{q-1} a_{k} \exp \lambda\left(z+\omega_{q}^{k} h\right)=\sum_{k=0}^{q-1} a_{k} \exp \omega_{q}^{k} h \lambda \exp \lambda z .
$$

Hence,

$$
\frac{A T_{h}(\exp \lambda z)}{\exp \lambda z}=\sum_{k=0}^{q-1} a_{k} \exp \omega_{q}^{k} h \lambda=A(\exp h \lambda) .
$$

On the other hand, by Property 1

$$
A T_{h}(\exp \lambda z)=\sum_{n=0}^{\infty} b_{n} \frac{h^{n}}{n !} D^{n} \exp \lambda z=\sum_{n=0}^{\infty} b_{n} \frac{h^{n} \lambda^{n}}{n !} \exp \lambda z .
$$

Then,

$$
\frac{A T_{h}(\exp \lambda z)}{\exp \lambda z}=\sum_{n=0}^{\infty} b_{n} \frac{h^{n} \lambda^{n}}{n !} .
$$

The property is proved.

Property 3. A q-sided shift operator $A T_{h}: O(\Omega) \rightarrow O\left(\Omega_{0}\right)$ is linear and continuous.

Proof. Linearity and continuity of the operator $A T_{h}: O(\Omega) \rightarrow O\left(\Omega_{0}\right)$ follow from the linearity and continuity of the shift operators $T_{\omega_{q}^{k} h}: O(\Omega) \rightarrow$ $O\left(\Omega_{0}\right), k \in\{0,1, \ldots, q-1\}$ and the representation

$$
A T_{h}(f)=\sum_{k=0}^{q-1} a_{k} T_{\omega_{q}^{k} h}(f) .
$$


The property is proved.

From Property 1 it follows that some of

$$
b_{n}:=\sum_{k=0}^{q-1} a_{k} \omega_{q}^{k n}, n \in\{0,1, \ldots\}
$$

are not equal to zero. Note that for any $n \in \mathbf{Z}_{+}$we have

$$
b_{n+q}=b_{n} .
$$

Hence, some of $b_{0}, \ldots, b_{q-1}$ are not equal to zero. The determinant

$$
\Delta_{0, \ldots, q-1}:=\left|\begin{array}{cccc}
\omega_{q}^{0} & \omega_{q}^{0} & \ldots & \omega_{q}^{0} \\
\omega_{q}^{0} & \omega_{q}^{1} & \ldots & \omega_{q}^{q-1} \\
\cdots & \cdots & \ldots & \cdots \\
\omega_{q}^{0} & \omega_{q}^{q-1} & \ldots & \omega_{q}^{(q-1)(q-1)}
\end{array}\right|=\prod_{0 \leqslant n<j \leqslant q-1}\left(\omega_{q}^{j}-\omega_{q}^{n}\right)
$$

of the system of equations

$$
\left\{\begin{array}{l}
a_{0}+a_{1}+\ldots+a_{q-1}=b_{0} \\
a_{0}+\omega_{q} a_{1}+\ldots+\omega_{q}^{q-1} a_{q-1}=b_{1} \\
\ldots \ldots \ldots \ldots \ldots \ldots \ldots \ldots \ldots \ldots \ldots \ldots \ldots \ldots \ldots \ldots \ldots \ldots \ldots \ldots \ldots \ldots \\
a_{0}+\omega_{q}^{q-1} a_{1}+\ldots+\omega_{q}^{(q-1)(q-1)} a_{q-1}=b_{q-1}
\end{array}\right.
$$

is not equal to zero. Thus, we obtain $a_{0}, \ldots, a_{q-1}$ from $b_{0}, \ldots, b_{q-1}$ :

$$
a_{k}=\frac{\Delta_{0, \ldots, q-1}^{k}\left(b_{0}, \ldots, b_{q-1}\right)}{\Delta_{0, \ldots, q-1}},
$$

where $\Delta_{0, \ldots, q-1}^{k}\left(b_{0}, \ldots, b_{q-1}\right)$ is the determinant

$$
\left|\begin{array}{ccccc}
\omega_{q}^{0} & \cdots & b_{0} & \cdots & \omega_{q}^{0} \\
\omega_{q}^{0} & \cdots & b_{1} & \cdots & \omega_{q}^{q-1} \\
\cdots & \cdots & \cdots & \cdots & \cdots \\
\omega_{q}^{0} & \cdots & b_{q-1} & \cdots & \omega_{q}^{(q-1)(q-1)}
\end{array}\right|
$$

obtained by replacing the $k$-th column by $\left(b_{0}, \ldots, b_{q-1}\right)^{T}$ in the determinant $\Delta_{0, \ldots, q-1}$.

Summarizing everything said above, we get the following property.

Property 4. A q-sided shift operator $A T_{h}$ is a differential operator of the form (3) such that: 
1) $b_{n}$ satisfy the condition (5);

2) some of $b_{0}, \ldots, b_{q-1}$ are not equal to zero.

The coefficients $a_{0}, \ldots, a_{q-1}$ are called the determining coefficients of the $q$-sided shift operator $A T_{h}$. The coefficients $b_{0}, \ldots, b_{q-1}$ are called the characteristic coefficients of the $q$-sided shift operator $A T_{h}$.

Denote by $n_{A}:=\left\{n_{1}, \ldots, n_{\nu}\right\}$ any ordered set of numbers $\{0, \ldots, q-1\}$ such that:

1) $0 \leqslant n_{1}<\ldots<n_{\nu} \leqslant q-1$

2) if $n \in\left\{n_{1}, \ldots, n_{\nu}\right\}$, then $b_{n} \neq 0$;

3) if $n \notin\left\{n_{1}, \ldots, n_{\nu}\right\}$, then $b_{n}=0$.

Such a set $n_{A}:=\left\{n_{1}, \ldots, n_{\nu}\right\}$ is called the indicator of the $q$-sided shift operator $A T_{h}$.

3. Exponential polynomials in some $D^{q}$-invariant space. Let $W$ be a $D^{q}$-invariant subspace of the space $O(\Omega)$. Any exponential polynomial that is in $W \subseteq O(\Omega)$ has the form

$$
\sum_{j=0}^{m} \sum_{k=0}^{q-1} p_{j, k}(z) \exp \left\{\omega_{q}^{k} \lambda_{j} z\right\}
$$

where $\lambda_{j} \in \mathbf{C}, p_{j, k}(z)$ are polynomials in $z, \omega_{q}:=\exp \frac{2 \pi i}{q}$. Since the exponential polynomial is in $W \subseteq O(\Omega)$, this polynomial is equal to the linear combination of special exponential polynomials.

Proposition 1. Any exponential polynomial from $W \subseteq O(\Omega)$ is equal to the linear combination of exponential monomials from $W$ that have the form

$$
\sum_{k=0}^{q-1} p_{k}(z) \exp \left\{\omega_{q}^{k} \lambda z\right\}
$$

where $\lambda \in \mathbf{C}, p_{k}(z)$ are polynomials in $z$.

Proof. Suppose that $\sigma(z) \in W$ and

$$
\sigma(z):=\sum_{j=0}^{m} \sum_{k=0}^{q-1} p_{j, k}(z) \exp \left\{\omega_{q}^{k} \lambda_{j} z\right\}, \lambda_{i} \neq \lambda_{j}, i \neq j .
$$


Let us act by the operator $D^{q}-\alpha$, where $\alpha \in \mathbf{C}$, on the exponential polynomial $\sigma(z)$; we get

$$
\left(D^{q}-\alpha\right) \sigma(z)=\sum_{j=0}^{m} \sum_{k=0}^{q-1} g_{j, k}(z) \exp \left\{\omega_{q}^{k} \lambda_{j} z\right\},
$$

where

$$
g_{j, k}(z)=\sum_{i=0}^{q}\left(\begin{array}{c}
q \\
i
\end{array}\right) \lambda_{j}^{q-1} \omega_{q}^{-i k} p_{j, k}^{(i)}(z)-\alpha p_{j, k}^{(i)}(z) .
$$

The following facts can be easily checked:

1) if $\lambda_{j}^{q}=\alpha$, then for any $k \in\{0, \ldots, q-1\} \operatorname{deg} g_{j, k}(z)<\operatorname{deg} p_{j, k}(z)$;

2) if $\lambda_{j}^{q} \neq \alpha$, then for any $k \in\{0, \ldots, q-1\} \operatorname{deg} p_{j, k}(z)=\operatorname{deg} g_{j, k}(z)$ and the leading coefficient of the polynomial $g_{j, k}(z)$ is equal to the leading coefficient of the polynomial $p_{j, k}(z)$ multiplied by $\lambda_{j}^{q}-\alpha$.

Let us act on $\sigma(z)$ by the operator

$$
\left(D^{q}-\lambda_{1}^{q}\right)^{n+1}\left(D^{q}-\lambda_{2}^{q}\right)^{n+1} \cdot \ldots \cdot\left(D^{q}-\lambda_{m}^{q}\right)^{n+1},
$$

where

$$
n=\max _{j, k}\left\{\operatorname{deg} p_{j, k}(z)\right\},(j, k) \in\{0, \ldots, m-1\} \times\{0, \ldots, q-1\} .
$$

By the above relations 1) and 2), we conclude that exponential polynomial

$$
\sigma_{0}(z):=\left(D^{q}-\lambda_{1}^{q}\right)^{n+1}\left(D^{q}-\lambda_{2}^{q}\right)^{n+1} \cdot \ldots \cdot\left(D^{q}-\lambda_{m}^{q}\right)^{n+1} \sigma(z)
$$

has the form

$$
\sum_{k=0}^{q-1} p_{k}(z) \exp \left\{\omega_{q}^{k} \lambda_{0} z\right\} .
$$

The leading coefficient of the polynomial $p_{k}(z)$ is equal to that of the polynomial $p_{0, k}$ multiplied by

$$
c_{0}:=\left(\lambda_{0}^{q}-\lambda_{1}^{q}\right)^{n+1}\left(\lambda_{0}^{q}-\lambda_{2}^{q}\right)^{n+1} \cdot \ldots \cdot\left(\lambda_{0}^{q}-\lambda_{m}^{q}\right)^{n+1} \neq 0 .
$$

Hence, the exponential polynomial

$$
\tilde{\sigma}(z):=\sigma(z)-c_{0} \sigma_{0}(z)
$$


has the form

$$
\tilde{\sigma}(z)=\sum_{j=0}^{m} \sum_{k=0}^{q-1} \tilde{p}_{j, k}(z) \exp \left\{\omega_{q}^{k} \lambda_{0} z\right\},
$$

where $\operatorname{deg} \tilde{p}_{0, k}(z)<\operatorname{deg} p_{0, k}(z)$ for any $k \in\{0, \ldots, q-1\}$.

Applying the above procedure to the exponential polynomial $\tilde{\sigma}(z)$ we continue to decrease the degrees of the polynomials $\tilde{p}_{0, k}(z)$. After that, we deal with the polynomials $p_{1, k}(z)$, etc. After a finite number of the steps we obtain:

$$
\sigma(z)=c_{0} \sigma_{0}(z)+c_{1} \sigma_{1}(z)+\ldots+c_{s} \sigma_{s}(z) .
$$

Note that the exponential polynomials $\sigma_{0}(z), \sigma_{1}(z), \ldots, \sigma_{s}(z)$ have the required form.

It is clear that any exponential polynomial of the form (7) has the representation

$$
e_{m}(z, \lambda):=\left.\frac{\partial^{m}}{\partial \zeta^{m}}\left(\sum_{k=0}^{q-1} g_{k}(\zeta) \exp \left\{\omega_{q}^{k} \zeta z\right\}\right)\right|_{\zeta=\lambda},
$$

where $\lambda \in \mathbf{C}, g_{k}(\zeta)$ are holomorphic at $\lambda$ functions.

Proposition 2. Any exponential polynomial from $W \subseteq O(\Omega)$ is equal to the linear combination of exponential monomials from $W$ that have the form (8).

Proposition 3. [3] If an exponential polynomial $e_{m}(z, \lambda)$ is in $W \subseteq O(\Omega)$ and $\lambda \neq 0$, then the exponential polynomials

$$
e_{0}(z, \lambda), e_{1}(z, \lambda), \ldots, e_{m-1}(z, \lambda)
$$

are in $W$.

4. Exponential polynomials and a $q$-sided shift operator. Choose an arbitrary exponential polynomial

$$
e_{m}(z, \lambda):=\left.\frac{\partial^{m}}{\partial \zeta^{m}}\left(\sum_{k=0}^{q-1} g_{k}(\zeta) \exp \left\{\omega_{q}^{k} \zeta z\right\}\right)\right|_{\zeta=\lambda}
$$


of the form (8). Then the $q$-sided shift operator

$$
A T_{h}: f(z) \rightarrow \sum_{k=0}^{q-1} a_{k} f\left(z+\omega_{q}^{k} h\right)
$$

(where $h \in U_{\varepsilon}$ is the shift) takes the exponential polynomial to

$$
A T_{h}\left(e_{m}(z, \lambda)\right):=\left.\frac{\partial^{m}}{\partial \zeta^{m}}\left(\sum_{k=0}^{q-1} g_{k}(\zeta) A T_{h}\left(\exp \left\{\omega_{q}^{k} \zeta z\right\}\right)\right)\right|_{\zeta=\lambda} .
$$

By Property 2 we have

$$
\begin{gathered}
A T_{h}\left(\exp \left\{\omega_{q}^{k} \zeta z\right\}\right)=A\left(\exp \left\{\omega_{q}^{k} \zeta h\right\}\right) \exp \left\{\omega_{q}^{k} \zeta z\right\}= \\
=\sum_{n=0}^{\infty} b_{n} \frac{\omega_{q}^{k n} \zeta^{n} h^{n}}{n !} \exp \left\{\omega_{q}^{k} \zeta z\right\},
\end{gathered}
$$

where $b_{n}$ are characteristic coefficients of the $q$-sided shift operator $A T_{h}$. Then,

$$
\begin{gathered}
A T_{h}\left(e_{m}(z, \lambda)\right)=\left.\frac{\partial^{m}}{\partial \zeta^{m}}\left(\sum_{k=0}^{q-1} g_{k}(\zeta) \sum_{n=0}^{\infty} b_{n} \frac{\omega_{q}^{k n} \zeta^{n} h^{n}}{n !} \exp \left\{\omega_{q}^{k} \zeta z\right\}\right)\right|_{\zeta=\lambda}= \\
=\left.\sum_{n=0}^{\infty} b_{n} \frac{h^{n}}{n !} \frac{\partial^{m}}{\partial \zeta^{m}}\left(\sum_{k=0}^{q-1} g_{k}(\zeta) \omega_{q}^{k n} \zeta^{n} \exp \left\{\omega_{q}^{k} \zeta z\right\}\right)\right|_{\zeta=\lambda}= \\
=\left.\sum_{n=0}^{\infty} b_{n} \frac{h^{n}}{n !} \frac{\partial^{m}}{\partial \zeta^{m}}\left(\zeta^{n} \sum_{k=0}^{q-1} g_{k}(\zeta) \omega_{q}^{k n} \exp \left\{\omega_{q}^{k} \zeta z\right\}\right)\right|_{\zeta=\lambda} .
\end{gathered}
$$

Choose an arbitrary functional $S \in O^{*}\left(\Omega_{0}\right)$; it takes the function $A T_{h}\left(e_{m}(z, \lambda)\right)$ to

$$
\begin{gathered}
\left\langle S, A T_{h}\left(e_{m}(z, \lambda)\right)\right\rangle= \\
=\left.\sum_{n=0}^{\infty} b_{n} \frac{h^{n}}{n !} \frac{\partial^{m}}{\partial \zeta^{m}}\left(\zeta^{n} \sum_{k=0}^{q-1} g_{k}(\zeta) \omega_{q}^{k n} \varphi\left(\omega_{q}^{k} \zeta\right)\right)\right|_{\zeta=\lambda},
\end{gathered}
$$

where $\varphi$ is a characteristic function of the functional $S$. 
Proposition 4. We obtain the equality

$$
\left\langle S, A T_{h}\left(e_{m}(z, \lambda)\right)\right\rangle=0
$$

for any $h \in U_{\varepsilon}$ iff functions

$$
b_{n}(\zeta)=: \zeta^{n} \sum_{k=0}^{q-1} g_{k}(\zeta) \omega_{q}^{k n} \varphi\left(\omega_{q}^{k} \zeta\right), n \in n_{A},
$$

are equal to zero at $\lambda$ with the multiplicity at least $m+1, n_{A}:=\left\{n_{1}, \ldots, n_{\nu}\right\}$ is the indicator of the $q$-sided shift operator $A T_{h}$.

Proof. Suppose that $\left\langle S, A T_{h}\left(e_{m}(z, \lambda)\right)\right\rangle=0$ for any $h \in U_{\varepsilon}$. Hence, $e_{m}(z, \lambda) \in W_{S} \subseteq O(\Omega)$, where $W_{S}$ is the solution space of the homogeneous $q$-sided convolution equation. $W_{S}$ is $D^{q}$-invariant. By Proposition 3 the exponential polynomials

$$
e_{0}(z, \lambda), e_{1}(z, \lambda), \ldots, e_{m-1}(z, \lambda)
$$

are in $W_{S}$. From (9) we have

$$
\begin{gathered}
\left\langle S, A T_{h}\left(e_{p}(z, \lambda)\right)\right\rangle= \\
=\left.\sum_{n=0}^{\infty} b_{n} \frac{h^{n}}{n !} \frac{\partial^{p}}{\partial \zeta^{p}}\left(\zeta^{n} \sum_{k=0}^{q-1} g_{k}(\zeta) \omega_{q}^{k n} \varphi\left(\omega_{q}^{k} \zeta\right)\right)\right|_{\zeta=\lambda}=0
\end{gathered}
$$

for any $h \in U_{\varepsilon}$ and $p \in\{0,1, \ldots, m\}$. It is clear that

$$
\left.\frac{\partial^{p}}{\partial \zeta^{p}}\left(\zeta^{n} \sum_{k=0}^{q-1} g_{k}(\zeta) \omega_{q}^{k n} \varphi\left(\omega_{q}^{k} \zeta\right)\right)\right|_{\zeta=\lambda}=0
$$

for any $p \in\{0,1, \ldots, m\}$ and $n \in n_{A}$. Thus, the functions $b_{n}(\zeta)$, where $n \in n_{A}$, have a zero $\lambda$ with the multiplicity at least $m+1$.

5. Elementary solutions of a homogeneous $q$-sided convolution equation. Now we obtain the description of the set consisting of exponential polynomials that are in the solution space $W_{S} \subseteq O(\Omega)$ of the homogeneous $q$-sided convolution equation (1). 
Denote by $\Delta_{0, \ldots, q-1}$ the Vandermonde determinant

$$
\left|\begin{array}{cccc}
\omega_{q}^{0} & \omega_{q}^{0} & \cdots & \omega_{q}^{0} \\
\omega_{q}^{0} & \omega_{q}^{1} & \cdots & \omega_{q}^{q-1} \\
\cdots & \cdots & \cdots & \cdots \\
\omega_{q}^{0} & \omega_{q}^{q-1} & \cdots & \omega_{q}^{(q-1)(q-1)}
\end{array}\right| .
$$

It is clear that

$$
\Delta_{0, \ldots, q-1}=\prod_{0 \leqslant j<i \leqslant q-1}\left(\omega_{q}^{i}-\omega_{q}^{j}\right) \neq 0 .
$$

Let $c_{0}(\zeta), c_{1}(\zeta), \ldots, c_{q-1}(\zeta)$ be a set of holomorphic at $\lambda \in \mathbf{C}$ functions. Denote by $\Delta_{k}\left(\zeta, c_{0}, \ldots, c_{q-1}\right)$ the determinant

$$
\begin{gathered}
\Delta_{0, \ldots, q-1}^{k}\left(c_{0}(\zeta), \frac{1}{\zeta} c_{1}(\zeta), \ldots, \frac{1}{\zeta^{q-1}} c_{q-1}(\zeta)\right)= \\
=\left(\begin{array}{ccccc}
\omega_{q}^{0} & \cdots & c_{0}(\zeta) & \cdots & \omega_{q}^{0} \\
\omega_{q}^{0} & \cdots & \frac{1}{\zeta} c_{1}(\zeta) & \cdots & \omega_{q}^{q-1} \\
\cdots & \cdots & \ldots & \cdots & \cdots \\
\omega_{q}^{0} & \cdots & \frac{1}{\zeta^{q-1}} c_{q-1}(\zeta) & \cdots & \omega_{q}^{(q-1)(q-1)}
\end{array}\right),
\end{gathered}
$$

that is obtained by replacing the $k$-th column in $\Delta_{0, \ldots, q-1}$ by

$$
\left(c_{0}(\zeta), \frac{1}{\zeta} c_{1}(\zeta), \ldots, \frac{1}{\zeta^{q-1}} c_{q-1}(\zeta)\right)^{T} .
$$

Theorem 1. Any elementary solution of a homogeneous q-sided convolution equation (1) is equal to a linear combination of exponential polynomials of the form

$$
e\left(z, c_{0}, \ldots, c_{q-1}\right):=\left.\frac{\partial^{m}}{\partial \zeta^{m}}\left(\sum_{k=0}^{q-1} \frac{\Delta_{k}\left(\zeta, c_{0}, \ldots, c_{q-1}\right)}{\varphi\left(\omega_{q}^{k} \zeta\right)} \exp \left\{\omega_{q}^{k} \zeta z\right\}\right)\right|_{\zeta=\lambda},
$$

where the holomorphic at $\lambda \in \mathbf{C}$ functions

$$
c_{0}:=c_{0}(\zeta), \ldots, c_{q-1}:=c_{q-1}(\zeta)
$$


and $m \in \mathbf{Z}_{+}$satisfy the following condition: functions

$$
\frac{\Delta_{k}\left(\zeta, c_{0}, \ldots, c_{q-1}\right)}{\varphi\left(\omega_{q}^{k} \zeta\right)}, k \in\{0,1, \ldots, q-1\}
$$

and functions

$$
\frac{c_{n}(\zeta)}{(\zeta-\lambda)^{m+1}}, n \in n_{A}
$$

are holomorphic at $\lambda$.

Proof. By Proposition 2, any exponential polynomial from a $D^{q}$-invariant $W_{S} \subseteq O(\Omega)$ is equal to the linear combination of exponential polynomials from $W_{S}$ of the form (8). Suppose that the exponential polynomial

$$
e_{m}(z, \lambda):=\left.\frac{\partial^{m}}{\partial \zeta^{m}}\left(\sum_{k=0}^{q-1} g_{k}(\zeta) \exp \left\{\omega_{q}^{k} \zeta z\right\}\right)\right|_{\zeta=\lambda}
$$

is in $W_{S} \subseteq O(\Omega)$. Suppose that $\varphi_{n}(\zeta):=\zeta^{n} \varphi(\zeta), n \in\{0,1, \ldots, q-1\}$. Then for any $n \in\{0,1, \ldots, q-1\}$ we have

$$
b_{n}(\zeta):=\zeta^{n} \sum_{k=0}^{q-1} g_{k}(\zeta) \omega_{q}^{k n} \varphi\left(\omega_{q}^{k} \zeta\right)=\sum_{k=0}^{q-1} g_{k}(\zeta) \varphi_{n}\left(\omega_{q}^{k} \zeta\right) .
$$

By Proposition 4, the functions $b_{n}(\zeta), n \in n_{A}$ have a zero $\lambda$ with the multiplicity at least $m+1$.

Consider the system of $q$ linear functional equations

$$
b_{n}(\zeta)=\sum_{k=0}^{q-1} g_{k}(\zeta) \varphi_{n}\left(\omega_{q}^{k} \zeta\right), n \in\{0,1, \ldots, q-1\},
$$

where $g_{k}(\zeta)$ are unknown holomorphic at $\lambda \in \mathbf{C}$ functions. The matrix of the system

$$
\left(\begin{array}{cccc}
\varphi_{0}\left(\omega_{q}^{0} \zeta\right) & \varphi_{0}\left(\omega_{q}^{1} \zeta\right) & \cdots & \varphi_{0}\left(\omega_{q}^{q-1} \zeta\right) \\
\varphi_{1}\left(\omega_{q}^{0} \zeta\right) & \varphi_{1}\left(\omega_{q}^{1} \zeta\right) & \cdots & \varphi_{1}\left(\omega_{q}^{q-1} \zeta\right) \\
\cdots & \cdots & \cdots & \cdots \\
\varphi_{q-1}\left(\omega_{q}^{0} \zeta\right) & \varphi_{q-1}\left(\omega_{q}^{1} \zeta\right) & \cdots & \varphi_{q-1}\left(\omega_{q}^{q-1} \zeta\right)
\end{array}\right)=
$$


$=\left(\begin{array}{cccc}\omega_{q}^{0} \zeta^{0} \varphi\left(\omega_{q}^{0} \zeta\right) & \omega_{q}^{0} \zeta^{0} \varphi\left(\omega_{q}^{1} \zeta\right) & \cdots & \omega_{q}^{0} \zeta^{0} \varphi\left(\omega_{q}^{q-1} \zeta\right) \\ \omega_{q}^{0} \zeta^{1} \varphi\left(\omega_{q}^{0} \zeta\right) & \omega_{q}^{1} \zeta^{1} \varphi\left(\omega_{q}^{1} \zeta\right) & \cdots & \omega_{q}^{q-1} \zeta^{1} \varphi\left(\omega_{q}^{q-1} \zeta\right) \\ \cdots & \cdots & \cdots & \cdots \\ \omega_{q}^{0} \zeta^{q-1} \varphi\left(\omega_{q}^{0} \zeta\right) & \omega_{q}^{q-1} \zeta^{q-1} \varphi\left(\omega_{q}^{1} \zeta\right) & \cdots & \omega_{q}^{(q-1)(q-1)} \zeta^{q-1} \varphi\left(\omega_{q}^{q-1} \zeta\right)\end{array}\right)$

has $q$ rows and $q$ columns. The determinant $\Delta_{0, \ldots, q-1}(\zeta \varphi(\zeta))$ of the matrix is equal to the following:

$$
\Delta_{0, \ldots, q-1} \zeta^{\frac{q(q-1)}{2}} \prod_{k=0}^{q-1} \varphi\left(\omega_{q}^{k} \zeta\right)
$$

where $\Delta_{0, \ldots, q-1}$ is the Vandermonde determinant

$$
\prod_{0 \leqslant j<i \leqslant q-1}\left(\omega_{q}^{i}-\omega_{q}^{j}\right) \neq 0
$$

Thus, the determinant $\Delta_{0, \ldots, q-1}(\zeta \varphi(\zeta))$ does not vanish in some neighborhood of $\lambda$, except $\lambda$ itself. By Cramer's rule

$$
g_{k}(\zeta)=\frac{\Delta_{0, \ldots, q-1}^{k}\left(\zeta \varphi(\zeta), b_{0}(\zeta), b_{1}(\zeta), \ldots, b_{q-1}(\zeta)\right)}{\Delta_{0, \ldots, q-1}(\zeta \varphi(\zeta))}, k \in\{0,1, \ldots, q-1\}
$$

where $\Delta_{0, \ldots, q-1}^{k}\left(\zeta \varphi(\zeta), b_{0}(\zeta), b_{1}(\zeta), \ldots, b_{q-1}(\zeta)\right)$ is the determinant

$$
\left(\begin{array}{ccccc}
\omega_{q}^{0} \zeta^{0} \varphi\left(\omega_{q}^{0} \zeta\right) & \ldots & b_{0}(\zeta) & \ldots & \omega_{q}^{0} \zeta^{0} \varphi\left(\omega_{q}^{q-1} \zeta\right) \\
\omega_{q}^{0} \zeta^{1} \varphi\left(\omega_{q}^{0} \zeta\right) & \ldots & b_{1}(\zeta) & \ldots & \omega_{q}^{q-1} \zeta^{1} \varphi\left(\omega_{q}^{q-1} \zeta\right) \\
\cdots & \ldots & \cdots & \cdots & \cdots \\
\omega_{q}^{0} \zeta^{q-1} \varphi\left(\omega_{q}^{0} \zeta\right) & \cdots & b_{q-1}(\zeta) & \cdots & \omega_{q}^{(q-1)(q-1)} \zeta^{q-1} \varphi\left(\omega_{q}^{q-1} \zeta\right)
\end{array}\right)
$$

that we obtain by replacing the $k$-th column in $\Delta_{0, \ldots, q-1}(\zeta \varphi(\zeta))$ by

$$
b_{0}(\zeta), b_{1}(\zeta), \ldots, b_{q-1}(\zeta)
$$

Then for any $k \in\{0,1, \ldots, q-1\}$ we have

$$
g_{k}(\zeta)=\frac{\Delta_{0, \ldots, q-1}^{k}\left(\zeta \varphi(\zeta), b_{0}(\zeta), b_{1}(\zeta), \ldots, b_{q-1}(\zeta)\right)}{\Delta_{0, \ldots, q-1}(\zeta \varphi(\zeta))}=\frac{\Delta_{k}\left(\zeta, c_{0}, \ldots, c_{q-1}\right)}{\varphi\left(\omega_{q}^{k} \zeta\right)}
$$


where

$$
c_{0}(\zeta):=\frac{b_{0}(\zeta)}{\Delta_{0, \ldots, q-1}}, \ldots, c_{q-1}(\zeta):=\frac{b_{q-1}(\zeta)}{\Delta_{0, \ldots, q-1}} .
$$

Thus the exponential polynomial $e_{m}(z, \lambda) \in W_{S}$ has a representation of the form

$$
\left.\frac{\partial^{m}}{\partial \zeta^{m}}\left(\sum_{k=0}^{q-1} \frac{\Delta_{k}\left(\zeta, c_{0}, \ldots, c_{q-1}\right)}{\varphi\left(\omega_{q}^{k} \zeta\right)} \exp \left\{\omega_{q}^{k} \zeta z\right\}\right)\right|_{\zeta=\lambda}
$$

and functions

$$
\begin{aligned}
\frac{\Delta_{k}\left(\zeta, c_{0}, \ldots, c_{q-1}\right)}{\varphi\left(\omega_{q}^{k} \zeta\right)} & =g_{k}(\zeta) \Delta_{0, \ldots, q-1}, k \in\{0,1, \ldots, q-1\}, \\
\frac{c_{n}(\zeta)}{(\zeta-\lambda)^{m+1}} & =\frac{b_{n}(\zeta)}{(\zeta-\lambda)^{m+1} \Delta_{0, \ldots, q-1}}, n \in n_{A}
\end{aligned}
$$

are holomorphic at $\lambda$.

Now consider an arbitrary exponential polynomial of the form

$$
e\left(z, c_{0}, \ldots, c_{q-1}\right):=\left.\frac{\partial^{m}}{\partial \zeta^{m}}\left(\sum_{k=0}^{q-1} \frac{\Delta_{k}\left(\zeta, c_{0}, \ldots, c_{q-1}\right)}{\varphi\left(\omega_{q}^{k} \zeta\right)} \exp \left\{\omega_{q}^{k} \zeta z\right\}\right)\right|_{\zeta=\lambda}
$$

where the holomorphic at $\lambda \in \mathbf{C}$ functions

$$
c_{0}:=c_{0}(\zeta), \ldots, c_{q-1}:=c_{q-1}(\zeta)
$$

and $m \in \mathbf{Z}_{+}$satisfy the following condition: the functions

$$
\frac{\Delta_{k}\left(\zeta, c_{0}, \ldots, c_{q-1}\right)}{\varphi\left(\omega_{q}^{k} \zeta\right)}, k \in\{0,1, \ldots, q-1\}
$$

and

$$
\frac{c_{n}(\zeta)}{(\zeta-\lambda)^{m+1}}, n \in n_{A}
$$

are holomorphic at $\lambda$. Let us show that $e\left(z, c_{0}, \ldots, c_{q-1}\right) \in W_{S}$. We need to prove that

$$
\left\langle S, A T_{h} e\left(z, c_{0}, \ldots, c_{q-1}\right)\right\rangle=0 .
$$

By Property 2 we have

$$
A T_{h}\left(e\left(z, c_{0}, \ldots, c_{q-1}\right)\right)=
$$




$$
\begin{gathered}
=\left.\frac{\partial^{m}}{\partial \zeta^{m}}\left(\sum_{k=0}^{q-1} \frac{\Delta_{k}\left(\zeta, c_{0}, \ldots, c_{q-1}\right)}{\varphi\left(\omega_{q}^{k} \zeta\right)} A T_{h}\left(\exp \left\{\omega_{q}^{k} \zeta z\right\}\right)\right)\right|_{\zeta=\lambda}= \\
=\left.\frac{\partial^{m}}{\partial \zeta^{m}}\left(\sum_{k=0}^{q-1} \frac{\Delta_{k}\left(\zeta, c_{0}, \ldots, c_{q-1}\right)}{\varphi\left(\omega_{q}^{k} \zeta\right)} A\left(\exp \left\{\omega_{q}^{k} \zeta z\right\}\right) \exp \left\{\omega_{q}^{k} \zeta z\right\}\right)\right|_{\zeta=\lambda}= \\
=\left.\frac{\partial^{m}}{\partial \zeta^{m}}\left(\sum_{k=0}^{q-1} \frac{\Delta_{k}\left(\zeta, c_{0}, \ldots, c_{q-1}\right)}{\varphi\left(\omega_{q}^{k} \zeta\right)} \sum_{n=0}^{\infty} b_{n} \frac{\omega_{q}^{k n} \zeta^{n} h^{n}}{n !} \exp \left\{\omega_{q}^{k} \zeta z\right\}\right)\right|_{\zeta=\lambda} .
\end{gathered}
$$

Hence,

$$
\begin{gathered}
A T_{h}\left(e\left(z, c_{0}, \ldots, c_{q-1}\right)\right)= \\
=\left.\sum_{n=0}^{\infty} b_{n} \frac{h^{n}}{n !} \frac{\partial^{m}}{\partial \zeta^{m}}\left(\zeta^{n} \sum_{k=0}^{q-1} \frac{\Delta_{k}\left(\zeta, c_{0}, \ldots, c_{q-1}\right)}{\varphi\left(\omega_{q}^{k} \zeta\right)} \omega_{q}^{k n} \exp \left\{\omega_{q}^{k} \zeta z\right\}\right)\right|_{\zeta=\lambda} .
\end{gathered}
$$

Since the functional $S$ is continuous,

$$
\begin{gathered}
\left\langle S, A T_{h}\left(e\left(z, c_{0}, \ldots, c_{q-1}\right)\right)\right\rangle= \\
=\left.\sum_{n=0}^{\infty} b_{n} \frac{h^{n}}{n !} \frac{\partial^{m}}{\partial \zeta^{m}}\left(\zeta^{n} \sum_{k=0}^{q-1} \frac{\Delta_{k}\left(\zeta, c_{0}, \ldots, c_{q-1}\right)}{\varphi\left(\omega_{q}^{k} \zeta\right)} \omega_{q}^{k n} \varphi\left(\omega_{q}^{k} \zeta\right)\right)\right|_{\zeta=\lambda}= \\
=\left.\Delta_{0, \ldots, q-1} \sum_{n=0}^{\infty} b_{n} \frac{h^{n}}{n !} \frac{\partial^{m}}{\partial \zeta^{m}}\left(\zeta^{n} \sum_{k=0}^{q-1} \omega_{q}^{k n} \frac{\Delta_{k}\left(\zeta, c_{0}, \ldots, c_{q-1}\right)}{\Delta_{0, \ldots, q-1}}\right)\right|_{\zeta=\lambda} .
\end{gathered}
$$

By the relations (5)

$$
\begin{gathered}
\left\langle S, A T_{h}\left(e\left(z, c_{0}, \ldots, c_{q-1}\right)\right)\right\rangle= \\
=\left.\Delta_{0, \ldots, q-1} \sum_{n=0}^{\infty} b_{n^{\prime}} \frac{h^{n}}{n !} \frac{\partial^{m}}{\partial \zeta^{m}}\left(\zeta^{n} \sum_{k=0}^{q-1} \omega_{q}^{k n^{\prime}} \frac{\Delta_{k}\left(\zeta, c_{0}, \ldots, c_{q-1}\right)}{\Delta_{0, \ldots, q-1}}\right)\right|_{\zeta=\lambda},
\end{gathered}
$$

where $n^{\prime}$ is the remainder after division of $n$ by $q$. By definition of the determinant $\Delta_{k}\left(\zeta, c_{0}, \ldots, c_{q-1}\right)$, we obtain

$$
\sum_{k=0}^{q-1} \omega_{q}^{k n^{\prime}} \frac{\Delta_{k}\left(\zeta, c_{0}, \ldots, c_{q-1}\right)}{\Delta_{0, \ldots, q-1}}=\frac{c_{n^{\prime}}(\zeta)}{\zeta^{n^{\prime}}} .
$$


Then,

$$
\begin{gathered}
\left\langle S, A T_{h}\left(e\left(z, c_{0}, \ldots, c_{q-1}\right)\right)\right\rangle= \\
=\left.\Delta_{0, \ldots, q-1} \sum_{n=0}^{\infty} b_{n^{\prime}} \frac{h^{n}}{n !} \frac{\partial^{m}}{\partial \zeta^{m}}\left(\zeta^{n-n^{\prime}} c_{n^{\prime}}(\zeta)\right)\right|_{\zeta=\lambda},
\end{gathered}
$$

where $b_{n^{\prime}}=0$, if $n^{\prime} \notin n_{A}$. Note that $c_{n^{\prime}}(\lambda)=0, c_{n^{\prime}}^{\prime}(\lambda)=0, \ldots, c_{n^{\prime}}^{(m)}(\lambda)=$ $=0$, if $n^{\prime} \in n_{A}$. Hence,

$$
\left\langle S, A T_{h}\left(e\left(z, c_{0}, \ldots, c_{q-1}\right)\right)\right\rangle=0 .
$$

The theorem is proved.

Acknowledgment. We thank the anonymous referees for valuable comments. Besides, we would like to express our acknowledgment to professor A. B. Shishkin.

\section{References}

[1] Krasichkov-Ternovskii I. F. Invariant subspaces of analytic functions. I. Spectral analysis on convex regions. Mat. Sb. (N.S.), 1972, vol. 87 (129), no. 4, pp. 459-489. DOI: https://doi.org/SM1972v017n01ABEH001488

[2] Krasichkov-Ternovskii I. F. Invariant subspaces of analytic functions. II. Spectral synthesis of convex domains. Mat. Sb. (N.S.), 1972, vol. 88, no. 1, pp. 3-30. DOI: https://doi.org/SM1972v017n01ABEH001488

[3] Shishkin A. B. Spectral synthesis for an operator generated by multiplication by a power of the independent variable. Mat. Sb., 1991, vol. 182, no. 6, pp. 828 -848. DOI: https://doi.org/SM1992v073n01ABEH002542.

Received May 21, 2018.

In revised form, September 3, 2018.

Accepted September 3, 2018.

Published online September 18, 2018.
A. A. Tatarkin
Kuban State University
149 Stavropolskaya st., Krasnodar 350040, Russia
E-mail: tiamatory@gmail.com;
U. S. Saranchuk
Kuban State University
149 Stavropolskaya st., Krasnodar 350040, Russia
E-mail: 89182859942@mail.ru 